\title{
INVESTIGACIÓN ACCIÓN CON ESTUDIANTES DE INTEGRACIÓN SOCIAL. UNA EXPERIENCIA BASADA EN LA RELACIÓN EDUCATIVA
}

\author{
José Javier Navarro Pérez \\ Universidad de Valencia \\ María Esperanza Campos Pons \\ IES Veles $i$ Vents (Gandia) \\ Francesc Xavier Uceda i Maza \\ Universidad de Valencia
}

\begin{abstract}
RESUMEN: La complejidad generada por la crisis coyuntural que actualmente vivimos, ha motivado una eclosión de la participación ciudadana en el espacio colectivo. Ello exige una reacción positiva por parte de la comunidad educativa e implica una nueva estrategia para el cambio social desde el grupo clase, a partir de metodologías exploratorias como la investigación acción que exijan el dinamismo de los individuos. El presente estudio, además nos muestra las principales motivaciones de los estudiantes de formación profesional superior en Integración Social, acercándonos a su perfil descriptivo. Estos estudios aunque desconocidos para la mayoría, contemplan entre sus bases curriculares, favorecer el desarrollo autónomo de las personas con una clara alusión a la dinamización del espacio local (Arriaga, 2007). Impulsamos el presente trabajo apoyados en las propuestas de Navarro y Puig (2010) respecto la relación educativa, al señalar ésta como una herramienta capaz de vincular los contenidos curriculares a la esfera afectiva entre docentes y estudiantes, generando además un compromiso que favorece la interacción, posibilita la detección de problemáticas y el desarrollo de climas positivos en la realidad social, desde la experiencia del grupo aula.
\end{abstract}

PALABRAS CLAVE: Experiencias docentes, investigación acción en educación, participación social, relación educativa, estudios profesionales.

\section{ACTION RESEARCH WITH SOCIAL INTEGRATION STUDENTS. A RELATIONSHIP EDUCATION EXPERIENCE}

ABSTRACT: The crisis which we live, has prompted an explosion of citizen participation in the collective space. This requires a positive reaction by the 
educational community and involves a new strategy for social change from the class group, from exploratory and research methodologies that require the dynamic action of individuals. This work is also the main motivations and interests of the students of Higher Level Training Course in Social Integration with the intention of describing a profile and to him the contributions made by the educational relationship. These studies are unknown to most but between their curricular objectives, aims to develop the autonomy of people with a clear allusion to the dynamism in the local space (Arriaga, 2007). We encourage this methodology supported proposals Navarro and Puig (2010), in this respect by stating that the effectiveness of the curriculum are linked to the charisma and affection with which the educator connect achieve the group classroom learning are more effective when the commitment and involvement of teachers are associated with the objectives of the curriculum, since the proximity in the same plane enables the detection of risks, promotes interaction and potentially a key positive development climate projective transform reality and social from the classroom.

KEYWORDS: Teaching experiences, action research in education, social participation, relationship education, professional studies.

Recibido: 08/05/2013

Aceptado: 04/09/2013

\section{INTRODUCCIÓN}

El actual marco de titulaciones del Espacio Europeo de Educación Superior supone una evolución y reordenación de los estudios universitarios, que a su vez implica una actualización de los estudios de Formación Profesional. La Ley Orgánica 5/2002 de 19 de junio relativa a las Cualificaciones y la Formación Profesional garantiza el impulso hacia un nuevo marco de titulaciones.

El área de Servicios Sociales, tradicionalmente multidisciplinar, ha albergado un abanico de profesionales procedentes tanto de estudios universitarios como de otras categorías con menor cualificación (Lucena et al., 2011), cuya función se ha ido adaptando a los diferentes niveles de intervención, atención y gestión tanto de servicios como de programas y recursos asociados a estos. Durante la última década, el nuevo catálogo de títulos de formación profesional ha reconocido titulaciones profesionales con un perfil vinculado al trabajo en los Servicios Comunitarios. Caso concreto de la Familia Profesional de Servicios Socioculturales y a la Comunidad, espacio en el que se encuentra incluida la Formación Profesional de Grado Superior en Integración Social que nos ocupa.

El Ciclo Formativo de Integración Social tiene su punto de partida, al igual que otras enseñanzas de formación profesional, tras la reforma educativa de 1990. Durante un largo periodo, se cuestionó la inexistencia de enseñanzas profesionales técnicas vinculadas a estudios universitarios de Trabajo Social y Educación Social, tal como si existía para otras titulaciones. Esta ilusión utópica, halló su germen a través de la 
regulación del Real Decreto 2061/1995 y publicado en BOE el 24-2-1996 y cuyo currículum aparece en el Real Decreto 1267/1997 de 24 de julio y publicado posteriormente en BOE el 11-09-97.

En relación a ello, el perfil profesional del Integrador Social, enunciado en el RD 2061/1995, señala que la cualificación profesional deberá incidir "en la programación, organización, desarrollo y evaluación de actividades de integración social, valorando la información obtenida sobre cada caso y determinando y aplicando estrategias y técnicas oportunas para el desarrollo de su autonomía personal e inserción ocupacional, actuando en cualquier caso bajo supervisión general de Licenciados o Diplomados". Es decir, el campo de cultivo del Integrador Social queda circunscrito a los Servicios Sociales.

Para ello, la duración del título se establece en 1700 horas, dividido en dos cursos; el primero de ellos destinado a la formación en materias teóricas y en el que destacan asignaturas de Metodología en Intervención Social (160 horas) o Habilidades de Autonomía Personal y Social (225 horas), y el segundo y último curso dedicado a la realización de Prácticas pre-profesionales mediante una asignatura instrumental denominada Formación en Centros de Trabajo (FCT), la cual presenta una duración de 740 horas. A este sentido, un estudio elaborado por Cueto, González y Mato (2006: 10) para el Principado de Asturias, refería la existencia de "mejores oportunidades de empleo para los Técnicos superiores que para los Técnicos Medios", incidiendo en la notoriedad que para ello representa la Formación en Centros de Trabajo, posibilitando amplia experiencia (pre) laboral y un enfrentamiento real a los problemas. Estas experiencias positivas de tradición germana, tratan de priorizar al máximo esa relación teórico-práctica. Llegan con fuerza, tanto que se plantea la puesta en marcha para el curso 2013-2014 de un modelo que optimiza la formación práctica y que viene instrumentalizado por el RD 1529/2012, de 8 de noviembre y publicado en el BOE el 9-11-2012 por el que se desarrolla el contrato para la formación y el aprendizaje y se establecen las bases de la Formación Profesional Dual, como formación práctica y especializada en los centros de trabajo.

En este marco, será clave el papel del profesor, quien experimenta por medio del currículo, la efectividad en la práctica de las diferentes acciones educativas que ejecuta. La implementación será distinta dependiendo del protagonista y por ello surgen problemas relativos al equilibrio del curriculum. Así pues, en palabras de Stenhouse (1985: 285): "lo deseable en la innovación educativa consiste en que mejoremos nuestra capacidad de someter a crítica nuestra práctica a la luz de nuestros conocimientos, y nuestros conocimientos a la luz de la realidad social". Ello responde claramente a la necesidad de que el docente investigue sus prioridades para obtener los mejores resultados, agrupándolos en cuatro líneas argumentales: Autodesarrollo profesional, mejorar la práctica docente, optimizar la institución educativa e incrementar en la comunidad estudiantil competencias para aplicar a la realidad social.

La investigación acción se propone como método para lograr estos resultados. A su vez, la relación educativa vehicula en un mismo plano contenidos curriculares y capacidades afectivas entre docentes y estudiantes, como explicamos a continuación. 


\subsection{Una experiencia basada en la metodología de Investigación-Acción}

El actual modelo educativo, configurado acorde a las necesidades de la sociedad industrial y las formas de cultura profesional del pasado siglo, se halla en una crisis estructural progresiva que exige nuevas respuestas.

A través de sus experiencias, Kurt Lewin (1946) trató de aproximar la investigación a la acción para la transformación de la realidad humana. Para éste, la investigación acción emerge de la teoría psicosocial y propone armonizar teoría y práctica en investigación acción, a través de un análisis del contexto, que categorice prioridades y evalúe a posteriori las acciones transformadoras.

Así, en este proceso de desarrollo, la investigación acción se erige como metodología capaz de enriquecer las prácticas educativas a través del compromiso de la comunidad educativa, en un marco de actualización, progreso y evolución de los intereses colectivos. Como proceso, avala la existencia de diferentes niveles. El primero de ellos, asume los siguientes parámetros:

- La investigación acción constituye un método que sobrepasa el aula, ya que la práctica docente no está reducida a ella, y en este sentido queda reflejado como los estudiantes de integración social requieren la traslación práctica del curriculum.

- Atendiendo a los criterios de Osorio y Rubio (2012), la investigación acción constituye una matriz por la cual el profesorado habrá de reconstruir su conocimiento profesional atendiendo la discusión reflexiva de sus problemas y necesidades. Además este tipo de escenarios y relaciones posibilitan la elaboración de un conocimiento profesional dialógico, multidisciplinar y heterogéneo que incrementará su valor (Elboj y Puigvert, 2003).

- La investigación acción ha de indagar en la opción colectiva, ya que requiere un proceso de intercambio y discusión.

- Se trata en definitiva de un enfoque multidimensional que exige la sistematización para proceder a su análisis.

Un segundo nivel viene configurado por el hecho de que la investigación acción, exige la participación docente. Kemmis (2007) establece como rasgos más representativos de la investigación acción el hecho de constituirse sobre la base de metodologías participativas y colaborativas.

Recientemente, un equipo de investigadores de la Universidad de Harvard encabezado por Tracy et al., (2012), incidía en las sinergias que el docente es capaz de generar sobre sus alumnos recreando la expresión de contenidos críticos y reflexivos, que exige el consenso, diálogo, y participación de los miembros de la comunidad educativa.

Por último el tercer nivel, ha de asociarse directamente con la actividad investigadora del educador docente. Este concepto nos transporta a la idea de Muñoz, Quintero y Munévar, (2002), al plantear que la práctica educativo-docente constituye un objeto para el análisis, que a su vez encaja con las técnicas cualitativas.

Para el desarrollo de los citados niveles de la investigación acción, hallamos la relación educativa como instrumento que deriva de dos grandes tradiciones en inter- 
vención social: la relación de ayuda (acompañada de diversas fórmulas y enfoques, principalmente desde la psicología, pero también desde el trabajo social), y los modelos educativos (tradición de carácter más generalista derivada de la las corrientes pedagógicas). Desde nuestra óptica, esta herramienta de la intervención social, se aproxima más a un método que a una técnica, y constituye el principal "saber hacer" que forma parte del "saber ser" de la práctica docente.

El establecimiento inicial de la relación de ayuda por parte del educador, puede resultar complejo (Tarín y Navarro, 2006), siendo una constante enfrentarse a reacciones desproporcionadas, apáticas, agresivas, etc. Este escenario es representativo de la situación que se vive en los centros educativos y de la distancia que separa a docentes de alumnos. Acercar las miradas, conjugar los intereses colectivos y reconducir ciertas actitudes para optimizar las relaciones, se convierte pues en una estrategia compleja a la vez que seductora, que necesita el vínculo para dar una respuesta más comprensiva y real (Howe, 1997) a las diferentes fragilidades que rodean la convivencia.

Partimos de una premisa básica: las acciones encaminadas al aprendizaje, exigen al profesional unos conocimientos específicos sobre aspectos educativos, que denominamos "saber hacer", y un talante o carisma que definimos "saber ser". Ambos saberes forman un cuerpo interdependiente, ya que mientras el "saber hacer" requiere del conocimiento metodológico y del dominio de unas técnicas específicas ${ }^{1}$, el "saber ser" supone una extensión del rol docente que involucra la esfera de la implicación afectiva.

Así pues, el educador encuentra en las instituciones que desempeña su actividad docente, escenarios que le permiten desarrollar procesos de investigación (Melero, 2012). En este sentido, y apoyando los argumentos de Pomar (2001) entendemos que el docente debe ser y actuar como educador para acceder al mundo afectivo de sus alumnos, y aplicar con éxito los tributos de su saber especifico.

Entendemos pues, que la relación educativa se convierte en herramienta básica para cualquier acción socioeducativa que trate de optimizar las necesidades colectivas.

Así pues, el presente estudio trata describir las experiencias de investigación acción en diferentes centros públicos de Formación Profesional con especialidad en Integración Social de la Comunitat Valenciana. Con objeto de trasladar los resultados de la misma a esferas ajenas al aula, se constituyeron como objetivos de la investigación los siguientes:

- Observar los niveles de motivación hacia el aprendizaje de los alumnos del Ciclo Formativo de Grado Superior en Integración Social (en adelante CFGSIS).

- Conocer las características de los estudiantes para intervenir curricularmente sobre ellos, atendiendo tanto sus potencialidades como sus déficits.

- Favorecer la participación positiva y el protagonismo de los alumnos en su proceso de adquisición de conocimiento.

- Reflexionar sobre las propias prácticas docentes para la elaboración de un manual de buenas prácticas capaz de mejorar las actividades de relación y de participación de los estudiantes de los CFGSIS.

1. Nos referimos a conocer y saber manejar las estrategias de la relación educativa. 


\section{Diseño DE INVESTigación}

El diseño se aproximó a los parámetros de investigación acción de Bisquerra (1989), que responden a un contexto situacional, participativo e interactivo que implica el dinamismo de los sujetos -comunidad educativa-, reflexivo y auto-evaluativo durante el proceso y de transformación global. Se proyectó la experiencia con objeto de transferir las buenas prácticas a otros contextos del curriculum del integrador social. Tal como se ha explicado previamente, representado en tres fases: planteamiento de la situación y objetivos, recogida de información para establecer el diagnóstico, y evaluación-transformación.

Además, se planteó la admisión de docentes que por propia voluntad quisieran vincularse al proyecto. Para ellos, fue criterio básico, que recibieran dos sesiones formativas relacionadas con el desarrollo de la relación educativa y la gestión de las dinámicas de aula. Estas sesiones centraron la importancia de la tutoría grupal, en la traslación al aula de los presupuestos participativos ${ }^{2}$, versión adaptada por Acosta (2009) para la dinamización juvenil desde bases comunitarias. Es decir, formar a los docentes en capacidades motivacionales, mediadoras y creativas para trasportarlas al aula y que los estudiantes pudieran experimentar en ellas.

Asimismo, también se recordaron conceptos sencillos de la investigación acción como herramienta metodológica -fases, aspectos colaborativos...-.

En definitiva, se planteó primero realizar un análisis cuantitativo, para posteriormente confrontar las evidencias con metodologías cualitativas. Ello permitió obtener un diálogo entre la unidad y la diversidad, que Bourdieu (1999) calificó como el espacio social de los puntos de vista. Para lograr los objetivos, se proyectó una investigación de carácter mixto, que según Pereira (2011: 19) "permite la obtención de una mejor evidencia y comprensión de los fenómenos".

Finalmente, la triangulación de la información garantizó la coherencia desde distintas ópticas analíticas (Denzin, 1978).

2. El presupuesto participativo se trata de una práctica política pública aplicada desde 1989 en Porto Alegre (Brasil), promocionada por Olivio Dutra, alcalde de la ciudad por el Partido de los Trabajadores. Esta política puede definirse como un proceso de intervención directa, permanente, voluntaria y universal, en el cual la ciudadanía, junto al gobierno, delibera y decide qué obras y servicios se deberán ejecutar con una parte de los recursos públicos locales. Ello trasladado a la educación, trata emerger como una gran apuesta por ampliar y mejorar las dinámicas del aula, las relaciones entre el alumnado, las prioridades e intereses sobre un escenario constructivo, reflexivo y crítico... Ello implica, plantear una cuestión que trasciende al colectivo de estudiantes como una sola entidad; es decir, ¿qué, cómo y de qué manera organizamos el grupo aula para favorecer sinergias colectivas, desarrollar procesos productivos efectivos, maximizar los intereses con la intención de desarrollar el espíritu colectivo y de alcanzar los objetivos curriculares? Es decir, en qué se invierte más tiempo, que prácticas didácticas aplicar, qué actividades diseñar y en definitiva democratizar las relaciones del grupo Aula, legitimando las acciones compartidas entre el docente y los estudiantes para completar el curriculum. Aquí el docente, tiene un papel muy importante en la creatividad, la capacidad mediadora, la propuesta, seguimiento y evaluación de actividades seductoras que faciliten la relación e inciten a la participación. Se trata en definitiva, de que el docente pueda contagiar al alumno el saber hacer y el saber ser en un mismo nivel y que ello pueda ser trasladado a otras esferas de la vida cotidiana. 


\subsection{Muestra}

La muestra estuvo compuesta por 114 estudiantes del CFGSIS matriculados en tres Institutos de Educación Secundaria de las tres provincias de la Comunidad Valencia$n^{3}$. Los criterios de inclusión para formar parte de la muestra fueron los siguientes: haber elegido la realización del presente módulo de Formación Profesional como primera opción, estar cursando el primer año del título, no haber iniciado el periodo de prácticas profesionales, no haber trabajado bajo ninguna categoría profesional en el sector cooperativo o de inclusión social.

Además de los estudiantes se incluyeron un total de 23 docentes, repartidos en diferentes centros educativos ${ }^{4}$. A diferencia de los alumnos, los docentes no formaron parte de la muestra a efectos cuantitativos, pero sí cualitativos. Para los docentes, se planteó como criterio de inclusión, que los centros educativos de los que dependían dispusiesen de medios, estabilidad laboral y que al menos un porcentaje superior al $50 \%$ de los docentes del CFGSIS desease participar en el estudio.

\subsection{Instrumentos}

De manera acorde con los objetivos y fases citadas, se utilizó una metodología cuanti-cualitativa. En una primera etapa de planteamiento inicial y con el fin de conocer a la población y detectar sus necesidades y motivaciones, se pasó un pretest a los estudiantes elaborado ad hoc mediante preguntas tanto cerradas como abiertas que permitió identificar variables personales, familiares, psicosociales, educativas, comunitarias, etc. Con ello pasamos a una segunda fase, donde obtuvimos un marco referencial sobre las necesidades y propuestas para la intervención. Con posterioridad, ya en la fase de evaluación se pasó un test utilizando la técnica test-retest que permitió comparar el antes y después de la acción transformadora. Así mismo se realizó un cuestionario de satisfacción en el que el alumnado podía evaluar los cambios acontecidos, cuestionamientos sobre el profesorado, su evolución personal, la metodología utilizada así como su perspectiva del proyecto y la posibilidad de plantear sugerencias.

La investigación fue complementada con dos técnicas cualitativas: entrevistas focalizadas a alumnos y docentes con la intención de verificar y complementar los resultados obtenidos mediante técnicas cuantitativas. Asimismo, se realizó una observación de las dinámicas de aula mediante registro, categorizándose las unidades de análisis para yuxtaponer los resultados. Para complementar este proceso, se dispuso de las Memorias de los diferentes Centros Educativos y otros datos facilitados por la Conselleria de Educación, Cultura y Deporte de la Generalitat Valenciana.

En las entrevistas focalizadas se siguieron las orientaciones de Patton (2002), mediante la elaboración de un guión con los temas y contenidos para su abordaje. Posteriormente se combinaron las técnicas cualitativas con las cuantitativas, permitiendo la obtención de información contrastada para proceder a su sistematización.

3. 39 estudiantes de Valencia, 39 de Alicante y 36 de Castellón.

4. 11 docentes en Valencia, 8 en Alicante y 4 en Castellón. 
El tratamiento cualitativo se efectuó mediante el programa informático ATLAS. ti 6. La codificación requirió de un proceso de asignación de categorías, conceptos y códigos a segmentos o testimonios de información que actuaron como soporte de interés para los objetivos de la investigación. Las categorías principales se orientaron desde tres áreas: motivación e intereses, curriculum y metodologías didácticas. A partir de estas, se desarrollaron sub-categorías que denominamos circunstanciales, ya que complementaron a las primeras. Entre ellas, destacamos las interacciones y propuestas, las experiencias de aula, la participación y las áreas de transformación, y por último las relaciones afectivo-emotivas, la percepción colectiva y las vivencias cotidianas del contexto socioeducativo, etc. El tratamiento de la información se realizó a partir de dos planos: textual y semántico, que posibilitaron la construcción de redes y la lógica articulación discursiva a partir de los niveles de abstracción.

\section{Resultados Y SU Discusión}

Las evidencias derivadas del análisis muestran la prolongación de la relación educativa como metodología didáctico-afectiva. Hemos planteado los resultados separando estos en atención a la metodología utilizada. En el diseño de la investigación, se planteó primero realizar un análisis cuantitativo que ahora presentamos, para posteriormente confrontar los resultados con técnicas cualitativas. Bajo esta misma premisa, mostramos los hallazgos obtenidos.

Por otro lado, el proceso de investigación - acción se organiza como proceso continuo, a modo de objetivos-problema, recogida de datos-diagnóstico, diseño de propuesta de cambio, ejecución y evaluación-reorientación. La convergencia de técnicas y la presentación de las evidencias, nos permitió desarrollar acorde a los objetivos, un manual para las buenas prácticas.

\section{Resultados Cuantitativos}

Dado que planteamos el trabajo en tres fases, la primera de ellas, relativa al planteamiento de la situación nos dejó unos resultados pre-test ${ }^{5}$ que reflejaron cierto grado de desmotivación en los estudiantes, describiendo un bajo rendimiento académico, baja participación e interacción tanto en la dinámica de aula como en la institucional-educativa, elevados porcentajes de dificultad en la comprensión conceptual y dudas respecto la valoración y análisis de textos especializados y en la realización del trabajos autónomos.

Del mismo modo, las estrategias didácticas de los docentes para facilitar el aprendizaje fueron definidas como "poco estimulantes"; más de 3/4 partes del alumnado trataba de evadirse de la interacción con el profesor. Los estudiantes clasificaron estas prácticas, sometidos en su mayoría a los clásicos paradigmas que implicaban estudiar memorizando, estrategia poco recomendable actualmente ya que impide relacionar conceptos, separa los segmentos cognitivos y provoca un olvido cuasi fulminante de los conocimientos adquiridos.

5. Fueron obtenidos con anterioridad al desarrollo de las sesiones formativas realizadas a los docentes que voluntariamente quisieron participar del estudio. 


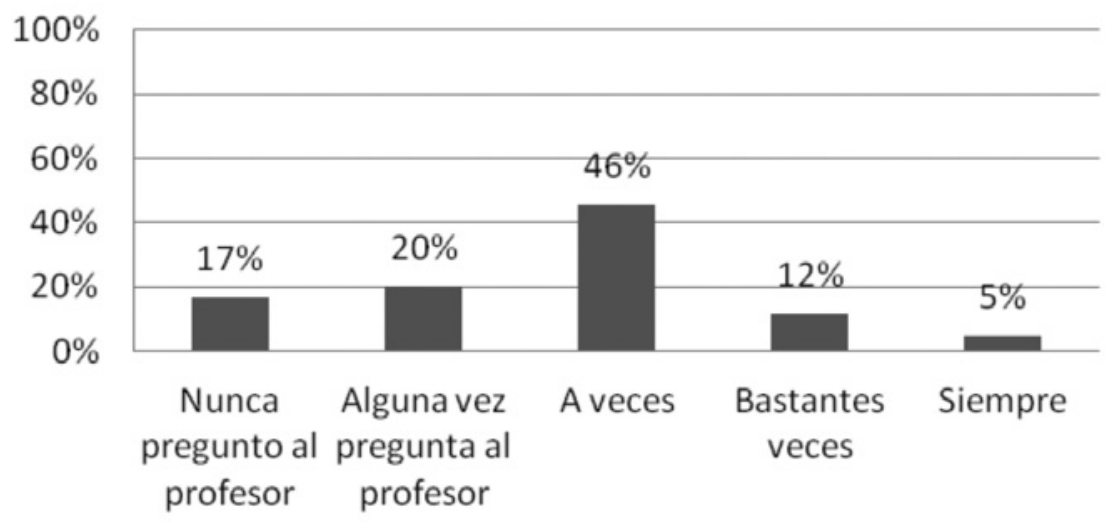

Fuente: Elaboración propia.

Figura 1. Participación de los estudiantes en el desarrollo de las clases

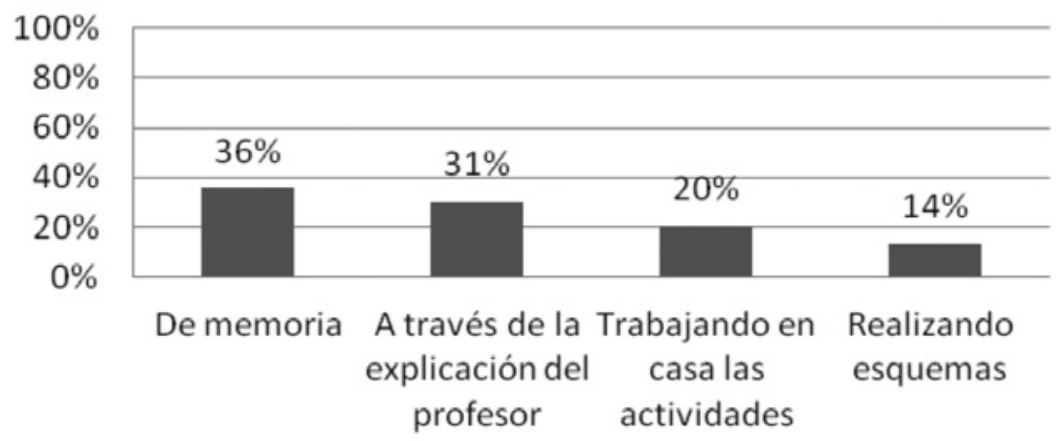

Fuente: Elaboración propia.

Figura 2. Clasificación según el estilo de Aprendizaje

Importantes fueron los hallazgos en las edades del alumnado sobre el que se desarrolló el estudio, ya que la madurez y los procesos de comprensión cognitiva formaron parte descriptiva del perfil. Así, derivado tanto de las fuentes secundarias como del cuestionario, obtuvimos unas características tipo de estudiante de CFGSIS que pasamos a describir:

Mayoría de estudiantes son mujeres (69\%), con una edad media de 24 años, desempleadas, que abandonaron los estudios para ocuparse en el mercado laboral, que han estado alejadas de la educación formal una media de 5 años, con pérdida de hábitos para el estudio y dificultades para integrar conceptos, que no establecen el proceso formativo como una prioridad sino como una actividad que evita la precarización o deterioro de su situación y que simultáneamente favorece la ampliación de 
competencias y que tienen interés o sensibilidad hacia la justicia social, la defensa y promoción de los más desfavorecidos, el interés por los derechos sociales y la implicación en movimientos solidarios.

Apoyados en otras fuentes secundarias consultadas, la presente muestra es ligeramente inferior porcentualmente a los datos aportados por Pérez Medina (2008) donde identificaba una proporción del $80 \%$ de mujeres matriculadas en esta especialidad. Por otro lado, coincidimos plenamente con el patrón descrito por el Consejo Escolar de Estado durante el curso 2010-2011, el cual refería que "el número de personas demandantes de estudios de Formación Profesional, se incrementó notablemente debido a la situación de desempleo en la que se encuentran y también a la falta de cualificación profesional adecuada, manteniendo dificultades en la integración a los nuevos estudios atendiendo la pérdida de hábitos y formación básica". A este respecto, durante la fase de recogida de información, observamos que los estudiantes que componen la muestra, describen el interés por la formación de manera provechosa, ya que según estos, los estudios conducentes a obtener el título de formación profesional en Grado Superior de Integración Social facilitan la inserción laboral. Vemos como un importante núcleo de la muestra (65\%) tiene una edad comprendida entre 21 - 28 años, es decir, en un elevado número de casos habían abandonado los estudios hace más de cinco años, presentando dificultades en la integración de contenidos competenciales de los que se compone el curriculum.

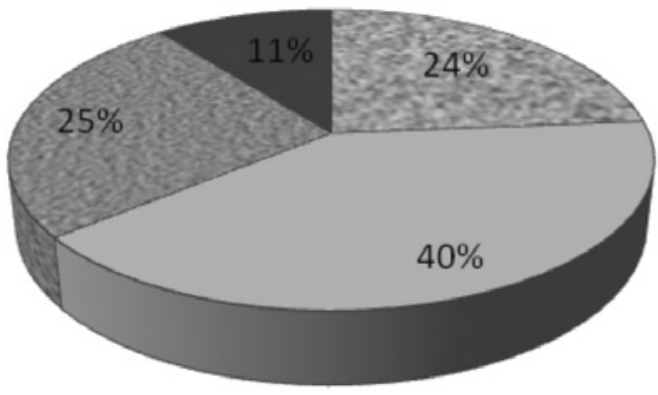

$\square 17-20$ años

$\square 21-24$ años

ㄴ25 - 28 años

Más de 28

años

Fuente: Elaboración propia.

Figura 3. Edad de los Estudiantes

Sin duda, que los resultados derivados de la recogida de información mediante pre-test nos obligaban a incentivar la relación educativa, ya que era capaz de aglutinar a través del "saber ser", estrategias del "saber hacer" favoreciendo la implicación y el protagonismo del alumnado. Estos ajustes requerían de un cambio de estilo pedagógico mediante el impulso de prácticas educativas consistentes, cercanas, seductoras y favorecedoras de la movilización colectiva. 
Así pues, se instó a los estudiantes a señalar propuestas participativas capaces de mejorar tanto las relaciones de aula, como fuera de ella pero vinculadas a los contenidos programados en el curriculum. Se instrumentalizó todo ello y los protagonistas (docentes y estudiantes) señalaron los principales déficits, realizaron propuestas de mejora y atendiendo a los planteamientos de Cuesta (2005), se trató de dar un giro tanto didáctico como de estilo, relacional, convivencial, proyectivo, etc. He aquí una muestra porcentual de las principales propuestas que se desarrollaron:

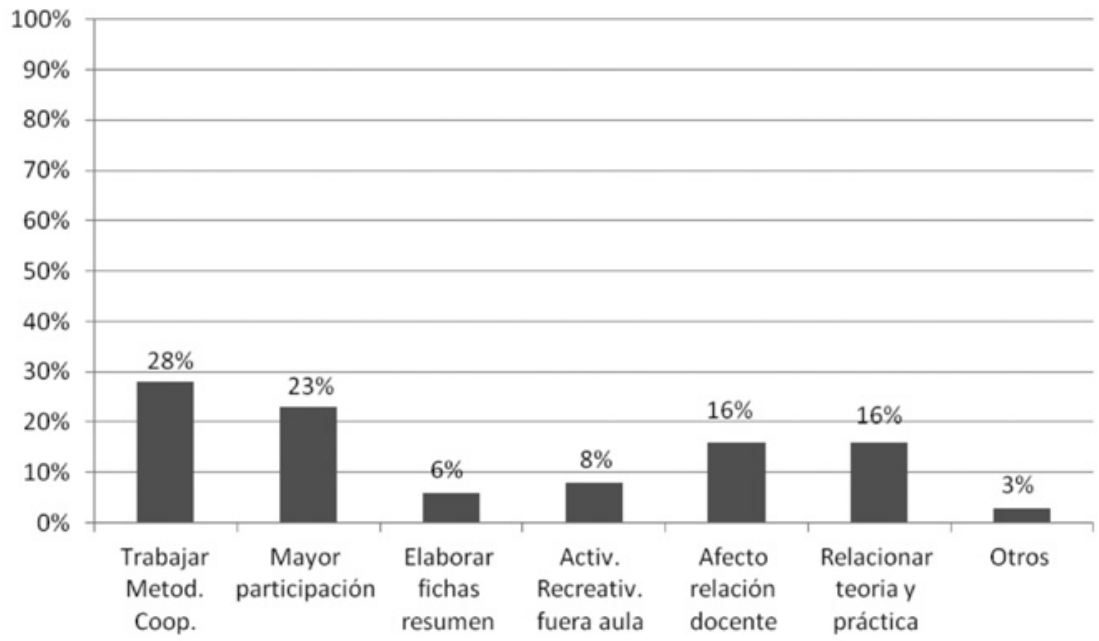

Fuente: Elaboración propia.

Figura 4. Propuestas de Mejora de la calidad educativa en el CFGSIS

La fase de evaluación, permitió mediante retest que los estudiantes señalaran dos sensibilidades bien delimitadas; por un lado, cambios en las áreas curriculares y por otro modificaciones en la percepción global. Así pues, relativo a la primera de ellas, identificaron un descenso en las dificultades al enfrentarse a los contenidos curriculares incluidos en la propia formación profesional. Destacar que las prácticas con más objeciones y críticas ${ }^{6}$, fueron dirigidas a la metodología de aprendizaje, la complejidad de los elementos teóricos, la gestión del trabajo autónomo en convergencia con la relación intergrupal. Esta fase de evaluación-reorientación permitió que Los argumentos planteados por estudiantes y profesores tuvieran un seguimiento mayor, liderado por la democratización de los presupuestos participativos, la adquisición de conceptos teóricos convergentes con las prácticas didácticas que incentivaron la gestión de nuevos contenidos, la motivación, el buen clima colaborativo, las experiencias comunitarias que se transportaron al aula y en definitiva, la percepción global caracterizada por el protagonismo del grupo aula hacia el aprendizaje autónomo.

6. Y que de alguna manera constituyeron el embrión que favoreció el cuestionamiento tanto de los métodos didácticos como de las estrategias para la adquisición de contenidos curriculares. 


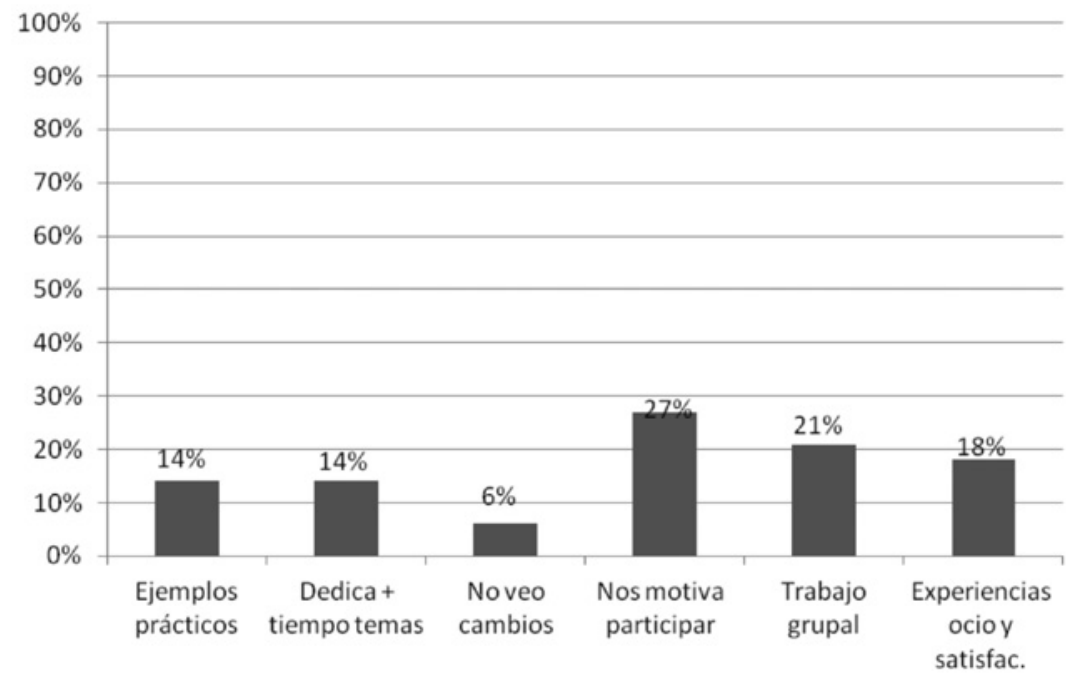

Fuente: Elaboración propia.

Figura 5. Cambios de primer orden en la percepción didáctica y curricular tras la intervención

Tal como encuadramos anteriormente, los estudiantes identificaron cambios que podríamos definir de segundo orden ${ }^{7}$. Establecemos como hallazgos no necesariamente vinculados a los contenidos curriculares ni a las actividades propias de aula, sino a la puesta en marcha de los presupuestos participativos que ampliaron las posibilidades de gestión del curriculum-aula.

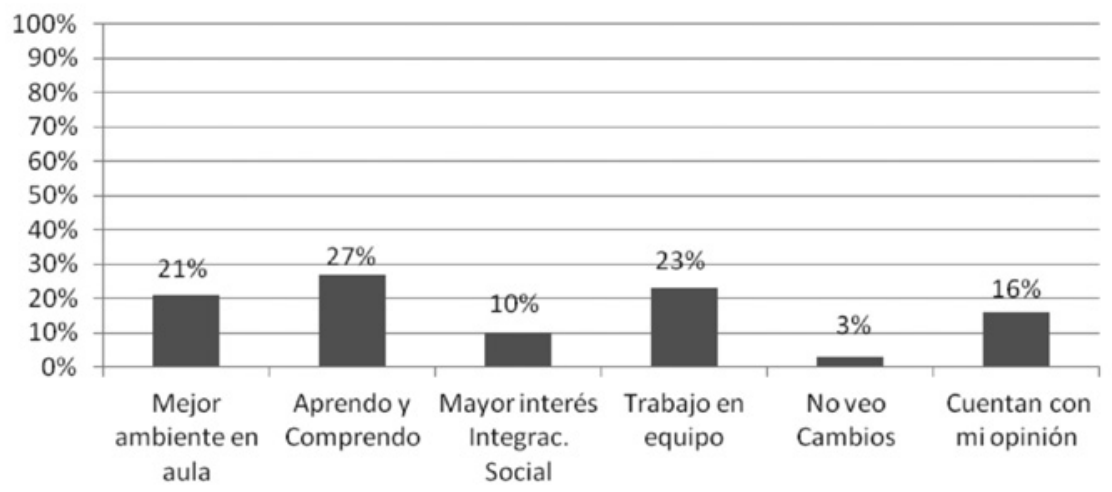

Fuente: Elaboración propia.

Figura 6. Cambios de segundo orden en la percepción global tras la intervención

7. O de un nivel quizá más global que conjugados con los anteriores ofrecieron mayor soporte. 


\section{Resultados Cualitativos}

Atendiendo a los objetivos planteados, los análisis derivados de la categorización de las observaciones y del análisis discursivo tras las entrevistas realizadas, sostienen las siguientes evidencias:

\section{El encorsetamiento curricular y los intereses del alumnado}

El planteamiento inicial previo a los objetivos, proyectó conocer los intereses del alumnado para incidir su motivación. En este sentido, hallamos que es el profesor quien guía y da una orientación constante, dejando poco espacio a la inercia y al protagonismo de los propios estudiantes. Los contenidos vienen limitados tanto por los objetivos curriculares como por las Guías que marcan la pauta, a modo de constante que asume la referencia de tutelar las diferentes acciones a lo largo del curso. No obstante, el mundo global en el que nos movemos, exige respuestas dinámicas que han de adaptarse a la práctica docente cotidiana.

se tiene que revisar todos los años, pero si la que tienes, la hiciste cuando Franco era cabo, pues ya dejamos de hablar... (DOC-Vlc1)

cada año cambian las cosas... Que si la ley de educación, la reforma laboral, ahora están con la ley de bases... tienes que reciclarte tú y después meter cositas por aquí y por allá [...]. (DOC-Cast2)

\section{Prácticas didácticas tradicionales}

La fase de diseño de objetivos, planteó como reto analizar las prácticas docentes. Desde este prisma, los resultados indican que los docentes utilizan en su mayoría prácticas didácticas basadas en aprendizajes memorísticos, que los alumnos desaprovechan para otros desempeños. Esto provocó en la mayor parte de los casos un alto índice de desmotivación (Fernández et al., 2010) y fracaso, al sentirse excluidos de un lugar normalizado (Cuesta, 2005).

Se te queda mejor algo que organizas tú y que te comes la cabeza para hacerlo o para organizarlo que el típico ejercicio que te viene en el libro y que te vas a la lectura de tal y lo sacas... (EST-1)

Si jo arribe a classe i dic, a vore obriu el Ilibre per la pàgina tal i comence ratatatata, ratatata, com si fora una metralladora, ¿ $\mathrm{m}^{\prime}$ entens?... està clar, demà no ve açí ni el tato ${ }^{8}$ (DOC-Vlc2)

para valorar lo que han aprendido es interesante fomentar debates, extender la crítica, hacer prácticas externas y después reflexionar en clase sobre lo que dicen unos y otros, y ponerlos en posiciones diferentes y con otros objetivos y experiencias ... (DOC-Alc2)

8. La traducción del valenciano es la siguiente: "si yo llego a clase y digo, a ver, abrid el libro por la página tal y empiezo ratatatata, ratatata, como si fuera una metralleta, ¿me entiendes?... claro, mañana no viene aquí ni el tato". 


\section{Obstrucción a la participación}

El segundo objetivo de la investigación trataba conocer el grado de participación en los centros escolares. En sentido los resultados informan de que ésta se considera baja y que incentivar actividades, favorece la creatividad, la improvisación y la recreación crítica de nuevos contenidos (Mejía, Orduz y Peralta, 2006), sin reducir la calidad de la docencia ni alterar los objetivos que diseña el curriculum. El sentimiento de clase como grupo, o como unión de miembros favorece las dinámicas participativas y cooperativas del alumnado entre sí.

Si un grupo es capaz de organizarse, ¿por qué no una clase entera? (DOC-Cast2)

Como en un tablero de ajedrez, es un ejército que cada uno tiene su oficio [...]. En clase, cada uno tiene su función y si no la asume, perjudica al resto (DOC-Alc1)

Los alumnos son capaces de percibir que el estilo y los métodos que desarrolla el docente en su estrategia didáctica, unidos a una organización rígida del aula constituyen las barreras más importantes que obstaculizan la participación en las actividades de formación.

haber estado tantos años interina, te hace conocer gente a la que el aula da seguridad, como si se resguardaran entre estas paredes (DOC-Alc6)

Así, las cualidades didácticas y educativas, ilustran la relación y las dinámicas del aula.

en la sala de profesores hay compañeros que dicen: ¡las cosas se hacen como yo digo!, porque luego no te agradecen nada y se creen en la obligación de [...]. Yo pienso que ni blanco ni negro, porque cuanto más motivados estén los chavales, tu trabajo será más satisfactorio (DOC-Cast2)

\section{¿Preparados para la vida "real"?}

La fase de recogida de información nos adentró en algunos aspectos que la entrevista cualitativa admite a diferencia de otras técnicas cuantitativas. Entendimos de utilidad, poder medir aspectos más profundos, la intensidad con la que el docente vive y comparte con sus alumnos las problemáticas sociales que acechan. Muy pocos docentes asumen con solvencia el tratamiento de estos problemas, por qué no definirlos de atención a la diversidad. Estas problemáticas pasan por encima de los contenidos curriculares, pues a la mella de estrategias didácticas y docentes se une el desgaste que llega a generar el conflicto de la precarización de las condiciones humanas. Además la integración social se aproxima a los márgenes de la realidad con mayor rigor y en ese sentido la distancia a la precariedad se precipita por mera inercia.

mira este año tengo varias chavalas que llevan todo el año como aquel que dice sin materiales. Ellas sin trabajo, sus parejas en el paro, algunas con criaturas pequeñas, viviendo con los padres y esto lo juntas con que hace un montón de años que dejaron de estudiar y ves que tienen la cabeza en otro sitio y esta variedad de situaciones nadie te enseña a cómo resolverlas y oye, hay que ser valiente y como yo les digo, sacar coraje y adelante" (DOC-Cast2) 
yo tengo 28 años y vivo situaciones en tutoría que me superan, ¿y por donde salgo? Estamos viviendo un momento muy difícil, pero hay que sacarlo entre todos como podamos (DOC-Alc2)

La situación actual sobrepasa la esfera personal. Lo positivo de estas cuestiones es que la dificultad actual exige un esfuerzo extra que permita enfrentarse a las debilidades de la vida real y orientarlas en la vida diaria.

\section{Docente y alumno ante el desafío de la convergencia}

La comunicación y las relaciones que se generan en el aula son muchas veces estresantes. La separación de roles profesional y personal, adultera la calidad humana que se ha de invertir en las aulas. Mucho más cuando la integración social como rama profesional, trata de ofrecer competencias a las personas que viven situaciones de precariedad para disponer de mejores herramientas para enfrentarse a las adversidades.

hay días que dices..., me abstengo a darles clase (DOC-Alc6)

yo intento ser siempre el mismo con todos pero siempre no sale bien. Esto es básico para detectar en qué punto están los chavales (DOC-VIc9)

El docente de esta especialidad tiene integrada esta dificultad en sus quehaceres cotidianos que exige la vida de aula y en muchas ocasiones traspasar esa barrera entre lo personal y lo profesional forma parte de una misma estructura. Si esta relación profesor-estudiantes, es capaz de trasladarse fuera de la esfera clase/aula, los mecanismos de ajuste social y de tutela del proceso educativo se extrapolan a otros espacios para la transformación. Así que, los presupuestos participativos, sirvieron de excelente técnica para la gestión de propuestas.

El que tu anomenes relació educativa ajuda a canviar els rols amb un plus que un tracte qualsevol no te dóna [...]. A voltes amb les empremtes que et deixen, pots endevinar situacions que es donaran avançant-te a d'elles ${ }^{9}$ (DOC-Vlc2)

Desde que empezamos el proyecto con vosotros, estamos colaborando con la plataforma de afectados por la Hipoteca. Una vez al mes hacemos una actividad con ellos y ayudamos con los materiales... cosas muy prácticas que es lo que ellos quieren y se involucran... (DOC-Cast2)

El intercambio comunicativo entre docentes y alumnos, repercute en una mejora del clima de la clase y en un mejor grado de satisfacción con la formación y los contenidos de la misma. Pero por otro lado, es fundamental identificar los riesgos añadidos a la mala gestión de las relaciones afectivas con los estudiantes y en la importancia que en ello adquiere el diálogo, la tutoría grupal e individual y el momento que vive el alumnado.

lo peor es confundir a la gente y a esto también hay que enseñarles. Con alumnos tan mayores la tutoría parece perder su interés, y realmente yo creo que te da más juego (DOC-Vlc1)

9. La traducción del valenciano es la siguiente: "lo que tu llamas relación educativa ayuda a cambiar roles con un plus que un trato cualquiera no te da... A veces con las huellas que te dejan, puedes adivinar situaciones adelantándote a ellas...". 
Observar el ritmo de aprendizaje de los alumnos y tratar de ajustarlo a la globalidad del aula, ensambla con las necesidades que expresan a menudo implícitamente los estudiantes.

cuando ves que uno no quiere, y se esconde... un reto, cuando hablan mucho pero lo importante se lo callan, otro, cuando aparecen y desaparecen, otro... mira si tenemos retos con los alumnos... (DOC-Alc1)

La investigación acción permitió realizar un diagnóstico para adaptar las necesidades objetivas a la realidad simbólica. La relación educativa y las actividades participativas redujeron las distancias afectivas entre docentes y estudiantes, y además legitimó la participación acorde a los objetivos curriculares. La estructuración en fases de investigación, permitió flexibilizar y reorientar constantemente el proceso.

\section{Conclusiones y propuestas}

El principal hallazgo que nos deja el presente estudio es la importancia que para el docente ha de representar el conocimiento del aula para asumir las inercias que en ella se generan; ello ayudará al docente a establecer diagnósticos fiables que posibiliten rediseñar los objetivos de aprendizaje.

Por otro lado, las posibilidades de la relación educativa amplían exponencialmente las relaciones con los estudiantes y ayudan en la dinamización de contenidos curriculares que la mera relación profesional no consigue abordar en su totalidad.

Las evidencias describen que la investigación acción combinada con la relación educativa y la instrumentalización de técnicas participativas, permite:

- Ajustar la temporalización al ritmo, interés y motivaciones del aula, bien modificando los contenidos o actividades de la unidad tomando en consideración las inquietudes de los estudiantes a efectos de introducir nuevas posibilidades.

- Responsabilizar a los estudiantes respecto sus propias elecciones; favoreciendo una participación más activa y dinámica en los compromisos adquiridos, generando nuevas situaciones en el diseño de propuestas de aprendizaje colectivas.

- Asimilar los contenidos con profundidad y bajo planteamientos críticos.

- Fortalecer las dinámicas de grupo mediante la relación educativa para desarrollar mayores y mejores competencias individuo-grupales.

También hemos de trasladar algunas dudas que se desprenden del presente análisis, el cual no está exento de limitaciones y, razonablemente, sus resultados han de ser interpretados con prudencia. Por otro lado, el trabajo plantea la necesidad de definir y fortalecer las relaciones entre los agentes que componen la comunidad educativa para su participación con la realidad local - social de la que forman parte. Además, la interdisciplinariedad profesional configurada actualmente desde los Servicios Sociales, focaliza más si cabe la atención en las prácticas grupales y cooperativas.

La estructuración por fases, ayudó tanto a alumnos como a docentes en la operatividad de sus acciones. La relación educativa promovió la eclosión de los presupuestos participativos. Transversalmente, los estudiantes se convirtieron en garantía 
legítima de su curriculum, vinculando la referencia del docente y su pertenencia al aula-grupo como materia indivisible en la dimensión social.

\section{ReFERENCIAS BibLIOGRÁfiCAS}

ACOSTA, A. (2009). Participación de la niñez y desarrollo local en un mundo globalizado. En Tonon, G., Comunidad, participación y socialización política. Buenos Aires: Espacio.

ARRIAGA, M. (2007). El entorno local como ámbito de acción socioeducativa. Zerbitzuan: Gizarte zerbitzuetarako aldizkaria, Revista de servicios sociales, 42, 61-71.

BISQUERRA, R. (1989). Métodos de investigación educativa. Guía práctica. Barcelona: CEAC.

BOURDIEU, P. (1999). La miseria del mundo. Madrid: Akal.

CUESTA, R. (2005). Felices y escolarizados. Crítica de la escuela en la era del capitalismo. Barcelona: Octaedro.

CUETO, B.; GONZÁLEZ, M.C. y MATO, F. J. (2006). El papel de la Formación en Centros de Trabajo (FCT) en la inserción laboral de los titulados de ciclos formativos: el caso de Asturias. Revista de educación, 341, ejemplar dedicado a: La cualificación profesional básica: competencias para la inclusión sociolaboral de jóvenes, 337-372.

DENZIN, N. (1978). The research act. A theoretical introduction to sociological methods. New York: McGraw-Hill.

ELBOJ, C y PUIGVERT, L. (2003). El giro dialógico de la sociología de la educación: aportaciones de la teoría sociológica a la sociología de la educación. Revista de gestión pública y privada, 8, 59-74.

FERNÁNDEZ, E.; MUÑOZ DE BUSTILLO, R.; BRAÑ, F. J. y ANTÓN, J. I. (2010). AIgunas apreciaciones aritméticas sobre el fracaso y el abandono escolar en España. Revista de educación, Extra 1, ejemplar dedicado a: Abandono temprano de la educación y la formación, 307-324.

HERNÁNDEZ, F. y TORT, A. (2009). Cambiar la mirada sobre el fracaso escolar desde la relación de los jóvenes con el saber. Revista Iberoamericana de Educación, 49, $8,1-11$.

HOWE, D. (1997). La teoría del vínculo afectivo para la práctica del trabajo social. Barcelona: Paidós.

KEMMIS, S. (2007). Sistema y mundo de vida, y las condiciones del aprendizaje en la modernidad. Kikiriki. Cooperación educativa, 82-83, 14-35.

LEWIN, K. (1946). Action research and minority problems. Journal of Social Issues, 2, 34-46.

LUCENA, M., ÁLVAREZ, J. y RODRÍGUEZ, C. (2011). Estudio de los valores para la empleabilidad en formación profesional. JETT, 2, (I), 21-27.

MEJÍA, J. A.; ORDUZ, M. y PERALTA, B. M. (2006). ¿Cómo formarnos para promover pensamiento crítico autónomo en el aula?: una propuesta de investigación acción apoyada por una herramienta conceptual. Revista Iberoamericana de Educación, 39, 6. Disponible en: http://www.rieoei.org/deloslectores/1499Mejia.pdf [Fecha de acceso: 24/04/2013]. 
MELERO, N. (2012). El paradigma crítico y los aportes de la investigación acción participativa en la transformación de la realidad: un análisis desde las ciencias sociales. Cuestiones pedagógicas: Revista de ciencias de la educación, 21, 339-355.

NAVARRO, J. J. y PUIG, M. (2010). El valor de la educación afectiva con niños en situación de vulnerabilidad acogidos en instituciones de protección: un modelo de Trabajo Social basado en la cotidianidad. Revista de Servicios Sociales y Política Social, 90, 65-84.

OSORIO, J. y RUBIO, G. (2012). Investigación-Acción desde un enfoque pedagógico eco-reflexivo: consideraciones para el desarrollo de un programa crítico-hermenéutico. Diálogos: Educación y formación de personas adultas, 69, 1, ejemplar dedicado a: Práctica y reflexión para una sociedad en cambio, 5-13.

PATTON, M. Q. (2002). Qualitative Research \& Evaluation Methods. Thousand Oaks, CA: Sage Publications.

PEREIRA, Z. (2011). Los diseños de método mixto de investigación en educación: una experiencia concreta. Revista Electrónica Educare, 25, 1, 15-29.

POMAR, M. I. (2001). El diálogo y la construcción compartida del saber. Barcelona: EUB.

STENHOUSE, L. (1985). Investigación y desarrollo del currículo. Madrid: Morata.

TARIN, M. y NAVARRO, J. J. (2006). Adolescentes en riesgo. Casos prácticos y estrategias de intervención socioeducativa. Madrid: CCS.

TRACY, E.; TRISHA, L. R.; SNOW, E. H. y SELMAN, R. S. (2012). Academic Discussions: An Analysis of Instructional Discourse and an Argument for an Integrative Assessment Framework. American Educational Research Journal, 49, (6), 12141250.

\section{Referencias Jurídico-Normativas}

Ley Orgánica 5/2002 de las Cualificaciones y de la Formación Profesional (BOE 2006-2002).

Real Decreto 2061/1995 de 22 de diciembre (BOE 24-02-1996).

Real Decreto 1267/1997 de 24 de julio (BOE 11-09-1997).

Real Decreto 1529/2012 de 8 de noviembre (BOE 9-11-2012).

Informe 2012 del Consejo Escolar del Estado relativo al estado del sistema educativo (Curso 2010-2011). Madrid: Ministerio de Educación, Cultura y Deporte, 2012. Disponible en: http://www.mecd.gob.es/dctm/cee/informe2012/i2012cee.pdf?do cumentld=0901e72b8145b4f2. [Fecha de acceso: 01/05/2013]. 